Swarthmore College

Works

2-1-1992

\title{
Light Scattering In The Isotropic Phase of Highly Chiral Liquid Crystals
}

\author{
J. E. Wyse
}

Peter J. Collings

Swarthmore College, pcollin1@swarthmore.edu

Follow this and additional works at: https://works.swarthmore.edu/fac-physics

Part of the Physics Commons

Let us know how access to these works benefits you

\section{Recommended Citation}

J. E. Wyse and Peter J. Collings. (1992). "Light Scattering In The Isotropic Phase of Highly Chiral Liquid Crystals". Physical Review A. Volume 45, Issue 4. 2449-2454. DOI: 10.1103/PhysRevA.45.2449 https://works.swarthmore.edu/fac-physics/223

This work is brought to you for free by Swarthmore College Libraries' Works. It has been accepted for inclusion in Physics \& Astronomy Faculty Works by an authorized administrator of Works. For more information, please contact myworks@swarthmore.edu. 


\title{
Light scattering in the isotropic phase of highly chiral liquid crystals
}

\author{
Joe E. Wyse and Peter J. Collings* \\ Department of Physics, Kenyon College, Gambier, Ohio 43022
}

(Received 28 May 1991)

\begin{abstract}
Light-scattering measurements using circularly polarized light in a backscattering geometry are employed to measure the amplitude of fluctuations in two of the five structural modes present in the isotropic phase of chiral liquid crystals. From these measurements, the second-order transition temperatures for all five modes are then calculated. In order to investigate the effect of chirality on the fluctuations, the experiments are performed in various mixtures of the chiral liquid crystal $4^{\prime \prime}$-(2-methylbutylphenyl)4'-(2-methylbutyl)-4-biphenylcarboxylate (CE2) and the nonchiral liquid crystal 4- $n$-pentylbenzenethio$4^{\prime}-n$-heptyloxybenzoate $(\overline{7} \mathrm{~S} 5)$. The results show that fluctuations in the five modes are independent in low-chirality mixtures, and, as predicted by theory, the second-order transition temperatures grow farther apart as the chirality increases. In highly chiral mixtures, fluctuations in the mode with the highest second-order transition temperature deviate from the normal temperature dependence, resulting in a lower second-order transition temperature for this mode. The probable explanation for this is that fluctuations in the structural modes are coupled in high-chirality systems, but at present no theoretical calculations exist.
\end{abstract}

PACS number(s): 61.30.-v, 64.70.Md, 78.20.Dj

\section{INTRODUCTION}

Significant progress in understanding both the phases which exist in highly chiral liquid crystals and the transitions between them has come from the realization that the order in chiral systems can be represented by a linear combination of five basic structural modes. The first theoretical work of this type was an investigation of the liquid-crystal-to-isotropic transition by Cheng and Meyer [1], which was later extended by Brazovskii and Dmitriev [2]. More recently, this approach proved to be extremely successful in explaining the nature of the blue phases [3]. Experimental verification of the theory as it relates to phase transitions was achieved mainly through opticalactivity measurements $[1,4]$, while optical-reflectivity experiments have been the most successful in confirming the theory of the blue phases $[5,6]$. Further theoretical work suggested that the optical activity in highly chiral systems might behave in a complex manner due to competing contributions from more than one structural mode [7], and this has been verified in a number of opticalactivity experiments [8-12].

Although light-scattering measurements should be just as revealing as optical-activity experiments in investigating these theoretical predictions, there has been much less work in this area. The light-scattering properties of chiral systems have been worked out by Hornreich and Shtrikman [13], but to date no experiments have been reported which make full use of these calculations. Bottger, et al. [14] conducted both static and dynamic light-scattering measurements on cholesteryl-oleylcarbonate (COC) using linearly polarized light and a scattering angle of $33^{\circ}$. Parthasarathy and DuPre [10] reported similar measurements on both pure COC and mixtures of COC with a nematic liquid crystal, using a scattering angle of $90^{\circ}$. Finally, Zink and Van Dael [15] performed measurements at various scattering angles between $30^{\circ}$ and $160^{\circ}$, using incident light which was either right circularly polarized (RCP) or left circularly polarized (LCP). While the results of these experiments gave some information on fluctuations in the various structural modes, the information was not as direct as it could be. As is shown in the following section, the most direct information on the fluctuations of these modes comes from backscattering $\left(180^{\circ}\right)$ experiments using circularly polarized incident light and detecting only circularly polarized light. Singh and Keyes have performed such an experiment on various cholesteryl esters, but the results have not yet been published [16]. None of the reported experiments utilized this feature of the theory.

After a short discussion of the theoretical considerations, we present the results of static light-scattering experiments which measure directly the amplitude of the fluctuations in two of the basic structural modes. By conducting the measurements in different mixtures of chiral 4"-(2-methylbutylphenyl)-4' -(2-methylbutyl)-4-biphenylcarboxylate (CE2) and nonchiral 4-n-penthylbenzenethio- $4^{\prime}-n$-heptyloxybenzoate ( $\left.\overline{7} \mathrm{~S} 5\right)$, the results show the effect of chirality on the fluctuations in these two modes. As predicted by the theory, the second-order transition temperatures for the five modes get farther apart as the chirality is increased. In mixtures of high chirality, the fluctuations in the mode with the highest second-order transition temperature are reduced slightly near the liquid-crystal transition, probably due to coupling between the structural modes.

\section{THEORY}

In the standard format of the Landau-de Gennes theory, the anisotropic part of the dielectric tensor $\epsilon_{i j}$ is used as the orientational order parameter. Since it is a 
symmetric tensor with zero trace, $\epsilon_{i j}$ has five independent elements. For chiral systems, it is appropriate to represent $\epsilon_{i j}$ as a linear combination of five basis tensors, each of which describes a different "structural mode" with a wave vector $q$. The five independent elements are the coefficients $\epsilon_{m}(q)$ of these five basis tensors. The $m= \pm 2$ modes are planar spiral modes where two principal axes are perpendicular to the spiral axis and rotate about it. The $m= \pm 1$ modes are conical spiral modes where two principal axes make an angle of $45^{\circ}$ to the spiral axis and rotate about it. The $m=0$ mode is a nematiclike mode, with no rotation of the principal axes. The handedness of the spiral is given by the sign of $m$, with the positive and negative sign denoting a left-handed and right-handed helix, respectively. In this representation, (i) a right-handed chiral nematic liquid crystal is described by a specific combination of the $m=0$ and -2 modes; and (ii) a right-handed chiral smectic- $C$ liquid crystal is described by a combination of the $m=0,-1$, and -2 modes, with the specific combination dependent on the tilt angle. In the latter case, an additional order parameter is necessary to describe the layering.

If the free energy is written as an expansion in $\epsilon_{i j}$ and its spatial derivatives (retaining terms up to second order), then each mode contributes independently to the free energy per unit volume $F(q)$,

$$
\begin{aligned}
F(q)=\frac{1}{2} \sum_{m}\left\{A-m B q_{0} q\right. \\
\left.+\left[B+(C / 6)\left(4-m^{2}\right)\right] q^{2}\right\}\left|\epsilon_{m}(q)\right|^{2} .
\end{aligned}
$$

$A, B$, and $C$, are coefficients in the expansion and $q_{0}$ is the chirality $4 \pi / P$, where $P$ is the pitch. The coefficient $A$ has the usual temperature dependence $A(T)$ $=A_{0}\left(T-T^{*}\right)$, where $T$ is the temperature and $T^{*}$ is the temperature at which a second-order transition would occur for a nonchiral system. By the equipartition theorem, the mean-square fluctuation of $\epsilon_{m}(q)$ in the isotropic phase is

$$
\begin{aligned}
\left\langle\left|\epsilon_{m}(q)\right|^{2}\right\rangle=\left(k_{B} T\right) /\{ & A-m B q_{0} q \\
+ & {\left.\left[B+(C / 6)\left(4-m^{2}\right)\right] q^{2}\right\}, }
\end{aligned}
$$

where $k_{B}$ is Boltzmann's constant. Since the denominator of Eq. (2) diverges at a different temperature depending on the value of $m$, each mode possesses a different second-order transition temperature,

$$
\begin{aligned}
& T_{ \pm 2}^{*}(q)=T^{*}+\left(B / A_{0}\right)\left( \pm 2 q_{0} q-q^{2}\right), \\
& T_{ \pm 1}^{*}(q)=T^{*}+\left(B / A_{0}\right)\left\{ \pm q_{0} q-[1+C /(2 B)] q^{2}\right\}, \\
& T_{0}^{*}(q)=T^{*}+\left(B / A_{0}\right)\left\{-[1+2 C /(3 B)] q^{2}\right\} .
\end{aligned}
$$

In Eq. (3), $q_{0}$ is assumed to be positive for a left-handed helix.

For typical values of $A_{0}, B, q_{0}$, and $q$, these five second-order transition temperatures differ by less than a degree even for highly chiral systems. Therefore fluctuations in all five modes will be appreciable in the isotropic phase. This is quite different from what occurs in liquidcrystal phases, where only certain modes exist. In that case, the fluctuations of one or two modes will dominate. In setting up an experiment to measure the fluctuations in a single mode in the isotropic phase, one must be careful to use a technique which is only sensitive to a single mode, since otherwise more than one mode will contribute.

The most direct way to measure the fluctuations in the isotropic phase is to perform light-scattering measurements. Such experiments are extremely powerful, since the wavelength of the light and the scattering geometry probe a unique value of $q$. Additionally, the polarization of the incident and detected light couple with the five modes in different ways, allowing one to select the mode under investigation. How the scattering geometry and polarization couple with the five modes has been worked out by Hornreich and Shtrikmann [13]. Using the Stokes vector and Mueller matrix formulation, they derive the scattering matrix in terms of the amplitude of the five modes. In general, the scattering matrix depends on all five modes, but in the case of backscattering, only the 0 and +2 modes are involved. If circularly polarized incident light is used and only circularly polarized light is detected in the backscattering geometry, then each of these three modes can be investigated individually. Specifically, if RCP light is incident and RCP light is detected, only fluctuations in the $m=-2$ mode contribute to backscattering. Similarly, using LCP incident light and detecting LCP backscattered light probes only fluctuations in the $m=2$ mode. Finally, having RCP light incident on the sample and detecting LCP which is backscattered is sensitive only to the $m=0$ mode, as is the opposite situation.

Previous light-scattering experiments did not take all of these considerations into account. The use of linearly polarized light or using circularly polarized light at scattering angles other than $180^{\circ}$ produces scattered light which is sensitive to the fluctuations in more then one mode, and in the isotropic phase no single mode dominates the scattering. The results presented here utilize the correct conditions for the investigation of single modes. Not only do these data reveal that the fluctuations in the modes with the highest and lowest secondorder transition temperatures are not that different in the isotropic phase, they allow the second-order transition temperatures for these two individual modes to be measured. By Eq. (3), the difference between the highest and lowest second-order transition temperatures for a righthanded system is given by

$$
T_{-2}^{*}(q)-T_{2}^{*}(q)=-\left(B / A_{0}\right)\left(4 q_{0} q\right) \text {. }
$$

This expectation is checked by measuring these two second-order transition temperatures for mixtures of varying chirality $q_{0}$.

It is also important to note that retaining higher than second-order terms in the free energy causes the structural modes to be coupled. In this case, fluctuations in one structural mode are no longer independent of fluctuations in the other modes. One would expect such a coupling to be important when the fluctuations are greatest. Thus evidence for such coupling should be seen (i) near the 
phase transition to the liquid-crystal phase, where the fluctuations in all of the modes are greatest; (ii) in properties dependent on the modes with the highest $T_{m}^{*}(q)$ temperatures, since these have the greatest fluctuations at all temperatures; and (iii) in highly chiral systems, where the phase transition temperature is close to the highest $T_{m}^{*}(q)$ temperatures and the resulting fluctuations are large [9].

It should also be pointed out that these second-order transition temperatures are for a specific value of $q$. This is appropriate for light-scattering measurements, where the experiment is sensitive to only one value of $q$. For theoretical purposes, the second-order transition temperatures for those values of $q$ which minimize the free energy of each mode are more important. These are closely related to the second-order transition temperatures given in Eq. (3) and are as follows [4]:

$$
\begin{aligned}
& T_{ \pm 2}^{*}=T^{*} \pm\left(B / A_{0}\right) q_{0}^{2}, \\
& T_{ \pm 1}^{*}=T^{*} \pm(1 /\{4[1+(C / 2 B)]\})\left(B / A_{0}\right) q_{0}^{2}, \\
& T_{0}^{*}=T^{*} .
\end{aligned}
$$

Since $q$ and $q_{0}$ are known, these second-order transition temperatures can be determined from the ones measured in a light-scattering experiment.

\section{EXPERIMENT}

The samples used in this experiment are mixtures of chiral CE2 and nonchiral $\overline{7} 55$, which have been described previously $[9,12,17]$. The pitch in the chiral nematic phase ranges from about $250 \mathrm{~nm}$ in the $40 \mathrm{wt}$ \% CE2 mixture to about $100 \mathrm{~nm}$ in pure CE2. The liquidcrystal-to-isotropic transition is different for each mixture, ranging from $92.4^{\circ} \mathrm{C}$ in the $40 \mathrm{wt} . \%$ CE2 mixture to $117.2^{\circ} \mathrm{C}$ in pure CE2. These mixtures have very high chirality, higher, for example, than the cholesteryl esters.

The experimental apparatus is quite simple. Using a lens with a long focal length, linearly polarized light from a 5-mW HeNe laser and $10 \times$ beam expander is focused on the sample through a quarter-wave plate oriented with its optic axis at $45^{\circ}$ to the direction of polarization. Light scattered backward from the sample passes through the same quarter-wave plate, is collected by another lens, and is focused through another polarizer onto a photomultiplier-tube detector placed to the side of the HeNe laser. The scattering angle is $170^{\circ}$. Two orientations of the quarter-wave plate are used. In one position, the light incident on the sample is RCP and only RCP leaving the sample passes through the polarizer in front of the detector. In the other position, LCP light is incident and only LCP scattered light makes it to the detector. Thus these two positions give information on the $m=-2$ and 2 modes, respectively. Two additional orientations of the polarizers (RCP incident and only LCP detected, and vice versa) give information on fluctuations of the $m=0$ mode, but the experiment is extremely difficult. Reflections from nonchiral materials (glass, for example) are detected in this arrangement, completely masking the weak signal from the liquid crystal. Even placing the sample at an angle does not reduce the interfering signal to a reasonable level.
The sample is contained between two pieces of glass separated by a $1-\mathrm{mm}$-thick spacer. This arrangement is placed in an Instec HS-1 hot stage, where the temperature is controlled to $\pm 0.001 \mathrm{~K}$. Since the light from the laser is focused to a very narrow beam at the sample, the temperature gradient across the part of the sample illuminated by the laser is extremely small, and certainly smaller than $0.01 \mathrm{~K}$. The data are taken by allowing the sample to come to equilibrium at each temperature, and then recording the number of counts in a given time period for each of the two positions of the quarter-wave plate. Data are taken with increasing temperature, starting at a temperature just below the transition in the liquid-crystal phase. The transition to the isotropic phase is clearly evident in the data, since the data in the liquid crystal phase show a slow, linear decrease with increasing temperature, while the data in the isotropic phase decrease rapidly and nonlinearly with increasing temperature. The measured transition temperatures are also consistent with optical-activity measurements made on the same system $[9,12,17]$. Measurements are made in 40,60 , 80 , and 100 wt. \% mixtures of CE2 in $7 \mathrm{~S} 5$.

According to Eq. (2), the scattered light intensity varies as $\left[T-T_{ \pm 2}^{*}(q)\right]^{-1}$ in the isotropic phase. The data are therefore best presented by graphing the reciprocal of intensity versus temperature, because then the data should be linear with the intercept along the temperature axis being $T_{ \pm 2}^{*}(q)$. This is done in Fig. 1 for the $40 \mathrm{wt}$. \% CE2 mixture and in Fig. 2 for pure CE2. The solid lines are least-squares fits to the expression

$$
I=K\left[T-T_{ \pm 2}^{*}(q)\right]^{-1}+I_{0},
$$

where $I$ is the backscattered intensity, and $K, T_{ \pm 2}^{*}(q)$, and $I_{0}$ are fitting parameters.

To determine how well the data follow Eq. (6), the fitting procedure is used repeatedly on the data, dropping

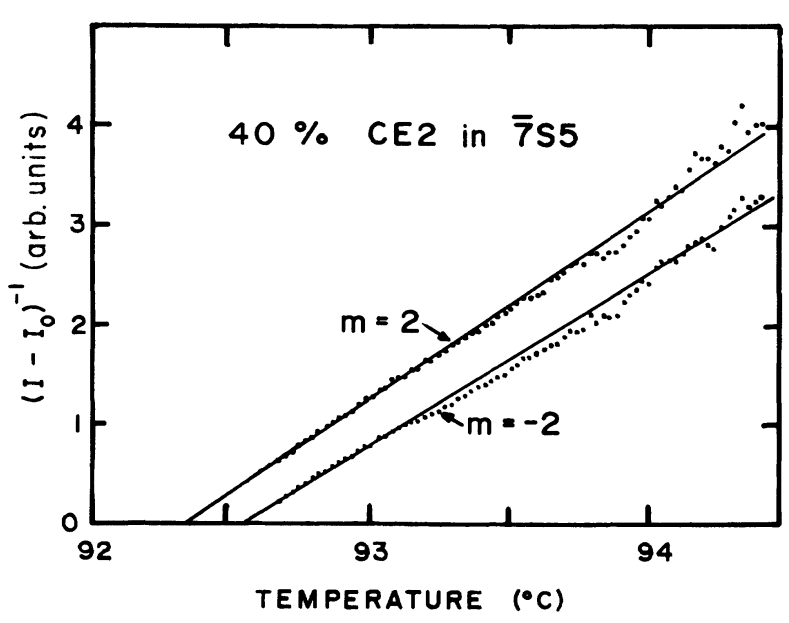

FIG. 1. Reciprocal of the backscattered intensity for 40 wt. \% CE2 in $\overline{7}$ S5. The units on the vertical axis are arbitrary. Data on the different modes are obtained using different orientations of the quarter-wave plate (see text). The solid lines are the least-squares fits to Eq. (6) using all of the data. 


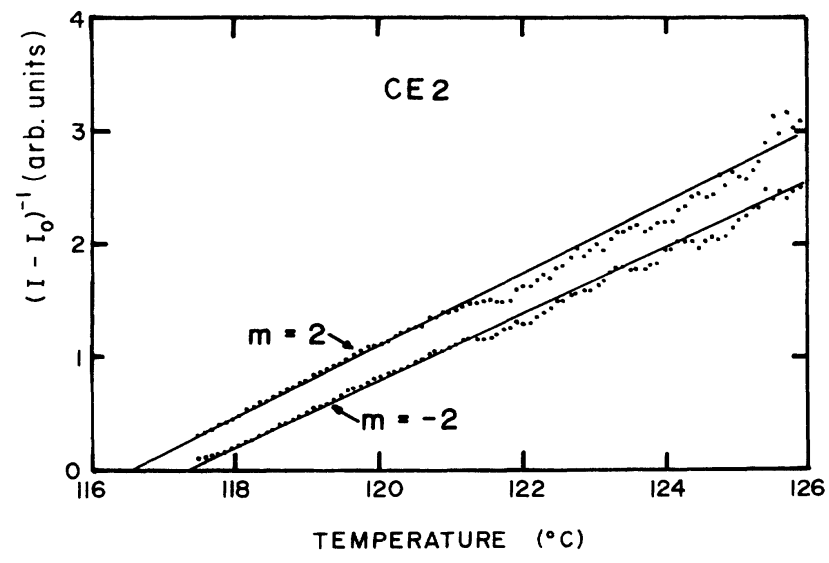

FIG. 2. Reciprocal of the backscattered intensity for pure CE2. The units on the vertical axis are arbitrary. Data on the different modes are obtained using different orientations of the quarter-wave plate (see text). The solid lines are the leastsquares fits to Eq. (6) using all of the data for the $m=2$ mode and the higher-temperature data for the $m=-2$ mode (see text).

the lowest temperature point each time. In the case of both sets of data for the 40 and 60 wt. \% CE2 mixtures and one set of data $(m=2)$ for the 80 and 100 wt. \% CE2 mixtures, this procedure produces no regular change to the fitting parameters. This is not the case for the $m=-2$ data set for the 80 and $100 \mathrm{wt} . \%$ CE2 mixtures. Here, dropping off the lowest data point causes a consistent change to the fitting parameters. The solid line in Fig. 2 for pure CE2 uses those values of the fitting parameters obtained after enough data points have been dropped so no further change is evident.

Theoretically, Eq. (4) states the difference between the highest and lowest second-order transition temperatures should be proportional to the chirality $q_{0}$, provided the other parameters remain constant. To a fairly good approximation, the chirality in these mixtures is proportional to the wt. \% of the chiral component. This difference in second-order transition temperatures is therefore graphed versus wt. \% CE2 in Fig. 3. Although

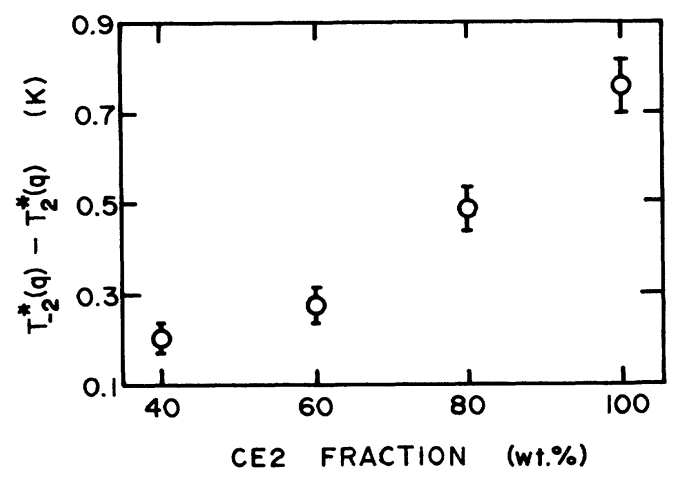

FIG. 3. Difference in the $m=-2$ and 2 second-order transition temperatures ( $q$ dependent) in mixtures with varying chirality.

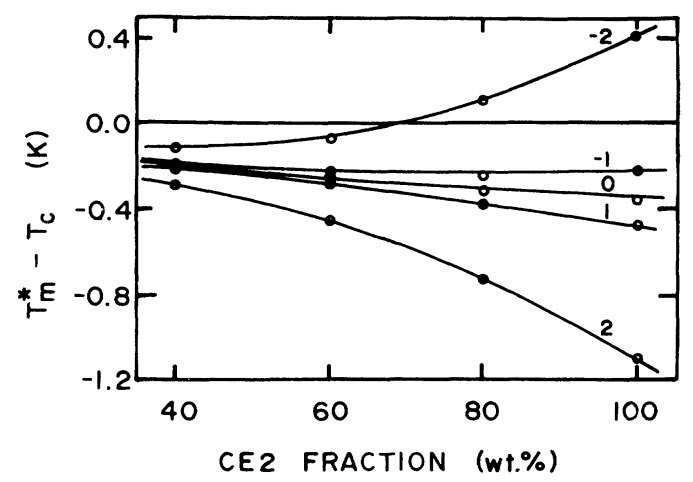

FIG. 4. Calculated $q$-independent second-order transition temperatures for all five modes $(m=0, \pm 1, \pm 2)$ in mixtures of varying chirality. $T_{c}$ is the liquid-crystal-to-isotropic transition temperature. The solid lines are least-squares quadratic fits to the data.

the data do not show a linear dependence, the increase in the temperature difference with increasing wt. \% CE2 is clearly evident. The deviation from linearity can easily be explained from the fact that the other parameters $\left(A_{0}\right.$ and $B$ ) vary as different amounts of CE2 and $\overline{7} S 5$ are mixed together.

Using the proper values for $q$ and $q_{0}$, the $q$ independent second-order transition temperatures of Eq. (5) are calculated for the four mixtures and graphed versus wt. \% CE2 in Fig. 4. One assumption is necessary, and that is that the coefficients $B$ and $C$ are about equal. There is good experimental evidence for this $[3,18]$, so the ratio $C / B$ is set to one in calculating the transition temperatures for Fig. 4. Even if this ratio deviates considerably from 1 , it changes only the $m= \pm 1$ temperatures slightly. To keep all of the data on the same graph, all temperatures are graphed relative to the liquid-crystal-to-isotropic transition temperature $T_{c}$. In mixtures of two optical isomers, the second-order transition temperatures of Eq. (5) should vary quadratically with $q_{0}$ (with no linear term). Since the measurements are not for mixtures of optical isomers and since all temperatures are relative to the transition temperature, it is not surprising that this simple dependence does not work in this case. Still, as evident from Fig. 4, quadratic fits (with a linear term) follow the data quite closely.

\section{DISCUSSION}

It first must be ascertained if a scattering angle of $170^{\circ}$ (rather than $180^{\circ}$ ) introduces fluctuations from other than the desired mode. Using the scattering matrix for a scattering angle of $170^{\circ}$, one finds (i) that scattering from the $m=0$ is multiplied by a factor of $10^{-6}$ from the desired mode, (ii) that the other $m= \pm 2$ mode contributes at the $10^{-11}$ level, and (iii) that the $m= \pm 1$ modes do not contribute at all. These numbers reveal that the real limitation to investigating the fluctuations in a single mode is experimental (quality and alignment of polarizers and quarter-wave plate), rather than the fact that a scattering angle of $170^{\circ}$ is used. In light of this, the polarizers and 
quarter-wave plate were carefully adjusted to ensure that circularly polarized light was being produced in the incident beam and detected by the photomultiplier tube.

The data for the lower chirality mixtures clearly support the theory. The light-scattering data follow the expected temperature dependence for the modes with the highest and lowest second-order transition temperatures. Only in the case of the higher-chirality mixtures does the data deviate from this dependence, and only for the mode with the highest second-order transition temperature. This occurs in a 1-K temperature region just above the transition to the liquid-crystal phase. The fact that this deviation occurs in the structural mode with the highest second-order transition temperature, near to the liquidcrystal transition, and only in high-chirality mixtures, indicates that coupling between the modes is a likely explanation.

It must also be pointed out that the second-order transition temperatures differ by less than a degree, even for the most highly chiral mixture. Thus for this system, the fluctuations in the "strongest" and "weakest" modes differ by at most a factor of 3 right above the transition, and less than this at higher temperatures. This makes it quite evident that all modes must be considered when performing experiments in the isotropic phase.

Figures 3 and 4 add even more support to the theory. While the exact theoretical dependence on chirality cannot be checked with these data, the results certainly show all the correct qualitative behavior. The data in Fig. 4 are the first to show the second-order transition temperatures for the five different modes and how they vary with chirality.

There is one other aspect of the data worthy of special attention. Notice that the highest $q$-independent secondorder transition temperature (as determined from the data away from the transition in the isotropic phase) is above the actual phase transition for the 80 and 100 wt. \% CE2 mixtures. These were the two mixtures that showed definite deviations from the uncoupled theory. Since the actual phase transition occurs at a higher temperature than all of the second-order transition temperatures, the second-order transition temperatures when coupling is important must be lower than for the uncoupled modes. This is evident from the data, since extrapolation of the data in the region where coupling is important gives a lower second-order transition temperature than if the rest of the data are used for the extrapolation. Therefore any theoretical attempt to describe the origin of the coupling must confront these experimental findings.

Finally, the values of the second-order transition temperatures shown in Fig. 4 are not quantitatively consistent with the measurements of $T_{-2}^{*}$ and $T_{-1}^{*}$ which result from optical-activity experiments $[9,12]$. Both types of measurements find that $T_{-2}^{*}$ increases relative to $T_{c}$ and that $T_{-1}^{*}$ decreases relative to $T_{c}$ with increasing chirality, but the magnitude of these changes differ be- tween the two experiments. Although they are quite consistent for the lowest-chirality mixture, the lightscattering results show that $T_{-2}^{*}$ increases more and that $T_{-1}^{*}$ decreases less as the chirality increases than reported for the optical-activity measurements. This discrepancy can be explained by the fact that the light-scattering results come from fits to the higher chirality data away from the region where the effects of coupling are present, while the fits to the optical activity data include this region using a theory for the optical activity which does not consider coupling. The optical activity theory does not account for the shift of the second-order transition temperatures (as the light-scattering data seems to imply), so fits which include this region produce lower values for the second-order transition temperatures. This would tend to decrease the apparent increase in $T_{-2}^{*}$ and increase the apparent decrease in $T_{-1}^{*}$ as the chirality is increased and coupling effects become stronger. This is in accord with the experimental results.

This type of behavior (but in the opposite direction) has been observed before in light-scattering experiments in the isotropic phase of compounds with a narrow nematic phase between the smectic and isotropic phases [19]. Coupling between fluctuations of the smectic and nematic order parameters causes the light-scattering intensity in a region near the nematic transition to deviate from its normal mean-field behavior. The effect of this coupling grows stronger as the width of the nematic phase decreases. A fit to the data in the temperature region where coupling effects are important gives a higher value for the second-order transition temperature than for the region where coupling is unimportant.

\section{CONCLUSION}

The light-scattering measurements reported here lend strong confirmation for the theory of independent structural modes in low-chirality systems and indicate that coupling between the structural modes is probably important in systems of high chirality. Not only are values of the five second-order transition temperatures reported here for the first time, but the data also suggest that coupling between the structural modes causes a shift of at least one of these second-order transition temperatures to a lower value.

\section{ACKNOWLEDGMENTS}

These results are based on work supported by the National Science Foundation (NSF) under Grants Nos. DMR-8906010 and DMR-9196048 and a William and Flora Hewlett Foundation grant from the Research Corporation (Grant No. C-2430). The authors thank P. Keyes for many useful discussions and C. Siders, G. Loveland, and J. Walker for important contributions to the experiments. 
*Present address: Department of Physics \& Astronomy, Swarthmore College, Swarthmore, PA 19081.

[1] J. Cheng and R. B. Meyer, Phys. Rev. Lett. 29, 1240 (1972); Phys. Rev. A 9, 2744 (1974).

[2] S. A. Brazovskii and S. G. Dmitreiv, Zh. Eksp. Teor. Fiz. 69, 979 (1975) [Sov. Phys. _JETP 42, 497 (1976)].

[3] H. Grebel, R. M. Hornreich, and S. Shtrikman, Phys. Rev. A 28, 1114 (1983); 30, 3264 (1984).

[4] V. K. Dolganov, S. P. Krylova, and V. M. Filev, Zh. Eksp. Teor. Fiz. 78, 2343 (1980) [Sov. Phys._JETP 51, 1177 (1980)].

[5] S. Meiboom and M. Sammon, Phys. Rev. Lett. 44, 882 (1980); Phys. Rev. A 24, 468 (1981).

[6] D. L. Johnson, J. H. Flack, and P. P. Crooker, Phys. Rev. Lett. 45, 641 (1980).

[7] V. M. Filev, Pis'ma Zh. Eksp. Teor. Fiz. 37, 589 (1983) [Sov. Phys. _ JETP Lett. 37, 703 (1983)].

[8] E. I. Demikhov and V. K. Dolganov, Pis'ma Zh. Eksp. Teor. Fiz. 38, 368 (1983) [Sov. Phys. - JETP Lett. 38, 445 (1983)].
[9] P. R. Battle, J. D. Miller, and P. J. Collings, Phys. Rev. A 36, 369 (1987).

[10] R. Parthasarathy and D. B. DuPre, Liq. Cryst. 3, 1231 (1988).

[11] F. Vanweert, W. Demol, and W. Van Dael, Liq. Cryst. 5, 853 (1989).

[12] J. E. Wyse, J. Ennis, and P. J. Collings, Phys. Rev. Lett. 62, 1045 (1989).

[13] R. M. Hornreich and S. Shtrikman, Phys. Rev. A 28, 1791 (1983).

[14] A. Bottger, L. Niessen, J. Coelingh, D. Frenkel, and R. Zijlstra, Liq. Cryst. 3, 337 (1988).

[15] H. Zink and W. Van Dael, Liq. Cryst. 5, 899 (1989).

[16] U. Singh and P. H. Keyes (private communication).

[17] J. Ennis, J. E. Wyse, and P. J. Collings, Liq. Cryst. 5, 861 (1989).

[18] T. W. Stinson and J. D. Litster, Phys. Rev. Lett. 30, 688 (1973).

[19] A Gohin, C. Destrade, H. Gasparoux, and J. Prost, J. Phys. (Paris) 44, 427 (1983). 